\title{
LA ÉTICA Y SU APORTE A LA CALIDAD QUE APLICA EL CONTADOR PÚBLICO EN EL PERÚ
}

\author{
ETHICS AND ITS CONTRIBUTION TO THE QUALITY THAT THE PUBLIC \\ ACCOUNTANT APPLIES IN PERU
}

\section{Ernesto A. Polar Falcón* / Sabino Talla Ramos / Percy A. Vílchez Olivares / Luis A. Angulo Silva/ Saturnino A. Ugarte Silva}

\author{
[Recepción: Abril de 2009 / Conformidad: Mayo de 2009]
}

\begin{abstract}
RESUMEN
Como difusión profesional, el presente artículo titulado: "La ética y su aporte a la calidad que aplica el contador público peruano", es un avance de la investigación que se está efectuando.; Muestra como inquietud preliminar, la necesidad de realizar estudios que faciliten conocer la relación que existe entre la ética profesional y el aporte a la calidad; se presentan conceptos y criterios de este importante tema, para que el lector se ubique profesionalmente y le permita evaluar los alcances de un Programa de Control de Calidad y sus aplicaciones. También se incluye un cuadro explicativo sobre la coordinación práctica que se tiene entre la ética profesional y el aporte a la calidad, que consolida una visión panorámica total del tema. Se muestra comparativamente las acciones de dos instituciones de Contadores Públicos, para despertar el interés en aportar una pronta aplicación de esta técnica en los trabajos profesionales de los Contadores Públicos en el Perú. Las conclusiones contienen mensajes positivos como colaboración a la profesión contable nacional.
\end{abstract}

Palabras clave: Normas de ética, aporte a la calidad, programa de control de calidad, coordinación práctica entre ética y aporte a la calidad.

\begin{abstract}
As a professional broadcast of this article entitled: "Ethics and its contribution to the quality that apply public peruvian" is part of the results of research being undertaken in the year 2009, showing concern as preliminary, the need facilitate studies of the relationship between professional ethics and contribution to the quality, concepts and approaches presented in this important topic so that the reader will fit professionally and able to assess of a Control Program Quality and its applications. Also included is an explanatory table which there is practical coordination between professional ethics and contribution to quality, which consolidates a total overview of the topic. Show the comparison of two measures of Certified Public Accountants, to arouse interest in making an early application of this technique in the work of professional accountants in Peru. To sum up contain positive messages as a collaborative national accounting profession.
\end{abstract}

Keywords: Rules of ethics, contribute to quality, quality control program, coordination between practical ethics and contribution to quality. * Doctor en Educación, Magíster en Contabilidad, Contador Público Colegiado Certificado, Docente Principal de la Facultad
de Ciencias Contables de la UNMSM. E-mail: epolarf@hotmail.com 
"Construir conocimiento científico, es aprender a pensar lo no pensado, lo cual supone un rompimiento de los modelos tradicionales, para atreverse a interdisciplinar ciencias entre sí para que actúen biounivocamente. La unión de la ética con la calidad es la mejor respuesta a la necesidad de sustentar este axioma para bien de los seres humanos". GASTÓN BACHELARD

\section{INTRODUCCIÓN}

El Trabajo de Investigación 2009, cuyo título está sirviendo para el presente artículo, contiene un tema de plena vigencia, tanto para América en general como para el Perú. Cuando se eligió este tema, todavía se escuchaban comentarios y se escribían libros sobre los problemas centrados principalmente en los casos ENRON y WORLD COM del año 2002 en USA, que conmovieron al mundo entero y afectaron a la profesión contable en América y en particular al Perú.

Las reacciones de protesta y análisis de esos problemas están plasmados en la buena cantidad de trabajos profesionales presentados en las Conferencias Interamericanas de Contabilidad (CIC), realizadas en los años 2005 y 2007, proponiendo inclusive fórmulas de soluciones.

En los Congresos Nacionales de Contadores Públicos del Perú, de los años 2004, 2006 y 2008, se han presentado algunos trabajos relacionados con esos problemas y también sobre la ética profesional. Solamente en el año 2006, se realizó la "I Convención Nacional de Ética y Ejercicio Profesional" con el lema de "Promoviendo la ética en la profesión contable y recuperando la confianza para una eficaz defensa profesional".

Entre el año 2002 y el año 2008, mucho se ha escrito sobre la ética profesional y las propuestas de cambios para mejorarla. Por ejemplo, en el Perú, en la profesión contable, se reformó el Código de Ética Profesional, aplicado tanto en forma individual como asociada. Lo que aún no ha tenido buena difusión es el tema de "la ética y su aporte a la calidad", del que en el presente artículo se muestran algunos avances de la investigación en curso.

\section{ALGUNOS CONCEPTOS Y CRITERIOS SOBRE LA ÉTICA Y SU APORTE A LA CALIDAD}

Para conocer mejor el enunciado de "La ética y su aporte a la calidad" se transcriben los siguientes conceptos y criterios ubicados en la investigación:

- "El desarrollo profesional del Contador Público no puede estar exento de la aplicación de normas éticas globalizadas acompañadas de normas de calidad adecuadas. De ninguna manera, se puede garantizar un servicio con valor agregado a los clientes digno de credibilidad o confiable, hecho por profesionales competentes, objetivos, responsables y que afronten los retos de los cambios acelerados en la profesión y que garanticen que la calidad de éstos sea indudable, sino se aplican los pronunciamientos profesionales de la comunidad contable sobre ética."

- "La fe pública que debe fortalecerse, recibe con las normas éticas y de calidad, los mejores ingredientes para asegurar que tal objetivo se genere $y$ que sea la premisa fundamental para el ejercicio profesional en condiciones óptimas."

- "La ética es el camino a seguir para conseguir el éxito y conviene mezclarla con la calidad, para obtener los beneficios que se buscan en el accionar de la profesión. Así, el Contador Público recibe la mejor recompensa, porque con este proceder se garantizan servicios de alto nivel. Desde el proceso de selección, su entrenamiento, supervisión durante el desempeño y su evalua- 
ción, están actuando fuerzas óptimas e insustituibles que son un imperativo social profesional, cuando ellas se colaboran entre sí (la ética y la calidad)."

- "Cuando se tiene el reto de conformar un código de ética que sirva a los intereses y al buen desempeño de la profesión a nivel continental, no se puede sustraer de éste, las normas de calidad, concebidas para obtener y conservar los clientes, asegurar confianza, formar profesionales exitosos. Dentro de estas normas de calidad, deben insertarse normas éticas."

- "El aporte de la ética a la calidad no nace solamente con la existencia de un código local o global. Se origina en el profesional en diferentes etapas de su desarrollo. Los esquemas académicos que lo van formando, el refuerzo a la ética como elemento clave a respetar durante el futuro ejercicio de su actividad, van contribuyendo a generar excelencia. Por ello, no es fácil definir con exactitud el momento en que hace su aporte la ética a la calidad. Pero, lo cierto es que todos esos aprendizajes, lo van modelando hacia en trabajo con adecuada calidad."

Después de los problemas contables del año 2002, la comunidad financiera está exigiendo a los contadores públicos que demuestren que el servicio que ofrecen, no solamente contenga criterios y conceptos de ética profesional, sino que se agregue un servicio de calidad. Frente a este requerimiento, se han planteado una serie programas de control relacionando la ética con la calidad. En el presente artículo, se va a mostrar un Programa de Control de Calidad, que otorga credibilidad y transparencia al trabajo que desarrolle el contador público.

Este Programa de Control de Calidad debe permitir la certificación de que el contador público:
1. Cumpla con la obligación profesional de establecer y mantener normas de calidad en el trabajo que realiza.

2. Promueva la calidad y eficiencia en la ejecución de los trabajos de conformidad con las normas profesionales.

3. Garantice el comportamiento íntegro y ético de su personal.

4. Desarrolle la participación en adiestramiento que fomente la preparación técnica y las cualidades profesionales, éticas y morales del personal.

Para verificar esta certificación y lograr una ejecución de alta calidad, se requiere contar con un buen sistema de monitoreo y un apropiado proceso educativo, a cargo de un revisor, que tenga a su cargo la preparación de un Informe sobre la idoneidad de las políticas de Control de Calidad en el trabajo o trabajos realizados. Esta revisión debe proveer al revisor:

1. Una base razonable para expresar una opinión sobre si el contador público ha demostrado la competencia necesaria para realizar los trabajos de contabilidad y auditoría, de acuerdo a las normas profesionales aplicables.

2. Evidencia suficiente para determinar si el sistema de control de calidad que el contador público ha diseñado está de acuerdo a los elementos de control de calidad adoptados por el organismo regulador correspondiente.

3. Elementos suficientes para evaluar si el sistema provee al contador público de una certeza razonable en cuanto al cumplimiento con las normas profesionales y los requisitos legales y de los reguladores, según le apliquen.

Este Programa de Control de Calidad tiene similitud de conceptos y enunciados con 
la Declaración de Práctica Profesional, de la Federación Internacional de Contadores (IFAC), titulada: "La calidad de los servicios profesionales".

En mayo del 2003, la Comisión Interamericana de Control de Calidad (CICC), aprobó el Programa Modelo de Control de Calidad de la Asociación Interamericana de Contabilidad (AIC), el cual fue ratificado por el Comité Ejecutivo en Panamá en setiembre del 2003. El Programa está dirigido a determinar si el sistema de control de calidad, diseñado para uso del contador público, está concordado de acuerdo a los elementos de control de calidad adoptados por la AIC y si dicho sistema provee al contador público de una certeza razonable en cuanto al cumplimiento con las normas profesionales. El
Programa Modelo entrará en vigencia al momento de ser aprobado por el Comité Ejecutivo de la AIC y luego de que se haya constituido la Junta Consultiva y de Supervisión y creado las estructuras operacionales y administrativas necesarias para administrarlo.

\section{COORDINACIÓN PRÁCTICA ENTRE LA ÉTICA Y SU APORTE A LA CALIDAD}

Como complemento a los enunciados teóricos que contiene este artículo, a continuación se muestra la necesaria coordinación que hay entre la ética y su aporte a la calidad, aplicables al desarrollo de un trabajo profesional:

\section{COORDINACIÓN PRÁCTICA ENTRE LAÉTICA Y SU APORTE A LA CALIDAD QUE APLICA EL CONTADOR PÚBLICO EN SU TRABAJO PROFESIONAL}

\begin{tabular}{|l|l|l|l|}
\hline \multicolumn{2}{|l|}{ NORMAS ÉTICAS COMUNES } & \multicolumn{2}{c|}{ APORTE A LA CALIDAD } \\
\hline 1. & Competencia & 1. & Trabajos de mejor nivel y aceptación \\
\hline 2. & Objetividad & 2. & Juicios y conceptos justos (Opinión) \\
\hline 3. & La Calidad & 3. & Es obvia \\
\hline 4. & Cuidado Personal & 4 & Mejor planeación, resultados exitosos, mejor servicio al cliente \\
\hline 5. & Planeación y Supervisión & 5. & Alcance adecuado de pruebas, eficiencia \\
\hline 6. & Cumplimiento de normas & 6. & Éxito y exoneración de sanciones personales y a los clientes \\
\hline 7. & Responsabilidad & 7. & Mejor presentación, mejor contenido, conclusiones adecuadas \\
\hline 8. & Confidencialidad & 8 & Exclusividad del trabajo en beneficio del interés de los clientes \\
\hline 9. & Diligencia & 9. & Oportunidad y mejores parámetros en los informes \\
\hline 10. & Independencia & 10. & Recomendaciones exitosas a los clientes \\
\hline 11. & Honorarios & 11. & Mayor esfuerzo y profundización en los trabajos \\
\hline 12. & Relaciones con otros Colegas & 12. & Alianzas exitosas en beneficio de la labor profesional \\
\hline 13. & Honestidad & 13. & Altos niveles de compromisos en la calidad \\
\hline 14 & Honorabilidad & 14. & Mejor aprecio por sus trabajos \\
\hline 15. & Lealtad & 15 & Mejor estimación a la calidad de su trabajo \\
\hline
\end{tabular}

LAÉTICAYSU APORTEALA CALIDAD A NIVEL PROFESIONAL EN EL PERÚ

Para lograr la excelencia de la profesión y optimizar la calidad de la labor realizada por los contadores públicos, el Colegio de Contadores Públicos Autorizados de Puerto Rico, cuenta desde el año 2001 con el Programa Voluntario de Control de Calidad, cuyo objetivo es mantener y mejorar la calidad de los servicios de contabilidad y auditoría que proveen las firmas de Contadores Públicos que participan en el Programa, que consta de tres secciones: 1) Informes Financieros; 
2) Consultoría, y 3) Revisión de Sistemas. Cuando una firma de contadores públicos o un contador público independiente se somete al proceso de revisión de calidad, demuestra su compromiso de ofrecer al público, servicios de contabilidad y auditoría que sean de calidad. Es decir, servicios en los que se puede confiar al hacer decisiones financieras, ya sean de negocios o personales.

En el Perú a nivel profesional se realizan limitadas aplicaciones de Ética y su Aporte a la Calidad. La crisis institucional que afecta al Colegio de Contadores Públicos de Lima está dañando la ética profesional de los contadores públicos y no permite desarrollar acciones para evaluar si se está cumpliendo con un adecuado aporte a la calidad, en beneficio de la comunidad financiera nacional.

\section{CONCLUSIONES}

1. El Código de Ética Profesional del Colegio de Contadores Públicos de Lima no tiene una difusión adecuada entre los miembros de la orden y no está actualizado frente a los requerimientos que exige el actual nivel de crecimiento económico que tiene el Perú y sus expectativas de crecimiento futuro, frente a la responsabilidad que asumen los Contadores Públicos en sus funciones públicas y privativas de ejercicio profesional.

2. A nivel institucional, se tiene una opinión general de que la profesión contable en el Perú, aplica con muchas limitaciones los conceptos, criterios y acciones necesarias para el uso de la ética y su aporte a la calidad, en los trabajos de contabilidad y auditoría, que actualmente se realizan, esperando que en un futuro a corto plazo, se desarrollen programas de estudios para asimilar estas técnicas que tienen tanta importancia profesional.

3. Desde el año 2006, en el Perú no se realiza evento alguno relacionado con la ética en general y de la ética y su aporte a la calidad en particular; no se difunden libros ni textos relacionados con estos temas; así como no se conocen los niveles éticos actuales que tienen los contadores públicos y los procesos de aplicación de estos temas, en sus trabajos profesionales de contabilidad y auditoría, tanto en el sector público como en el privado, frente a los problemas de la crisis internacional que afecta al desarrollo de la economía nacional.

\section{REFERENCIAS BIBLIOGRÁFICAS}

1. Canetti, Marcelo. Hacia la optimización del control de calidad. Argentina: AIC.

2. Emmi, Blanca. El contador público y el diseño de políticas públicas. Claves éticas a tener en cuenta. Uruguay: AIC.

3. Emmi, Blanca. Claves éticas para restaurar la confianza. Uruguay: AIC.

4. Freire, Liliana Beatriz. Ética e independencia en el ejercicio profesional. En busca de la confianza perdida. Argentina: AIC.

5. Grisola, Héctor Julio. Ética y responsabilidad social en el ejercicio profesional del contador público. Argentina: AIC.

6. IFAC. Control de calidad en el trabajo de auditoría. México: AIC.

7. León Gómez, Jaime. La ética y la calidad. Colombia: AIC. 
8. Panez Meza, Julio. El control de calidad en prácticas de contabilidad y auditoría. Perú: AIC.

8. Ramírez, Elisa Eva. Los contadores públicos y el Estado: Recuperar la confianza para enfrentar la corrupción. Argentina: AIC.

9. Santos Jesús, Flor. Conciencia e independencia profesional. Argentina: AIC.

10. Vitta, C. M. La educación continuada.
Norma profesional obligatoria necesaria para garantizar un servicio de calidad. AIC, Argentina.

11. Washigton, Diego. Mecanismos para restaurar la confianza en la profesión. AIC, Argentina.

12. Zaá, José Rafael. Fundamentos éticos coadyuvantes para el mantenimiento de la independencia del contador público en el ejercicio profesional. 